\author{
Applicable Analysis and Discrete Mathematics \\ available online at http://pefmath.etf.rs
}

Appl. Anal. Discrete Math. 5 (2011), 165-175. doi:10.2298/Aadm110909022C

\title{
CONTROLLABLE GRAPHS WITH LEAST \\ EIGENVALUE AT LEAST -2
}

\author{
Dragoš Cvetković, Peter Rowlinson, \\ Zoran Stanić, Myung-Gon Yoon
}

\begin{abstract}
Connected graphs whose eigenvalues are distinct and main are called controllable graphs in view of certain applications in control theory. We give some general characterizations of the controllable graphs whose least eigenvalue is bounded from below by -2 ; in particular, we determine all the controllable exceptional graphs. We also investigate the controllable graphs whose second largest eigenvalue does not exceed 1.
\end{abstract}

\section{INTRODUCTION}

Let $G$ be a simple graph with $n$ vertices and adjacency matrix $A(=A(G))$. The eigenvalues of $A$ are also called the eigenvalues of $G$. These eigenvalues, in non-increasing order, are denoted by $\lambda_{1}\left(=\lambda_{1}(G)\right), \ldots, \lambda_{n}\left(=\lambda_{n}(G)\right)$.

An eigenvalue of a graph is called main if the corresponding eigenspace contains a vector for which the sum of coordinates is different from 0 , while the connected graphs in which all eigenvalues are mutually distinct and main are called controllable (see $[\mathbf{7}]$ and $[\mathbf{9}]$ ). Note that a connected graph with $n$ vertices is controllable if and only if it has no eigenvector orthogonal to the all-1 vector $\mathbf{j}_{n} \in \mathbb{R}^{n}$ (a property which first ensures that each eigenspace has dimension 1).

We recall from [7] an application of controllable graphs in control theory that explains their name. The following differential equation is a standard model for physical systems:

$$
\frac{\mathrm{d} \mathbf{x}}{\mathrm{d} t}=A \mathbf{x}+\mathbf{b} u .
$$

2010 Mathematics Subject Classification. 05C50

Keywords and Phrases. Graph spectra, control theory, main eigenvalues, bounded eigenvalues. 
Here $\mathbf{x}=\mathbf{x}(t)$ is called the state vector, with given $\mathbf{x}(0)$, and the scalar $u=u(t)$ is the control input. The matrix $A$ has size $n \times n$, while both $\mathbf{x}$ and $\mathbf{b}$ have size $n \times 1$.

The above system is called controllable if the following is true: given any vector $\mathbf{x}^{*}$ and time $t^{*}$, there always exists a control function $u(t), 0<t<t^{*}$, such that the solution of (1) gives $\mathbf{x}\left(t^{*}\right)=\mathbf{x}^{*}$ irrespective of $\mathbf{x}(0)$. That is, the state can be steered to any point of $n$-dimensional vector space arbitrarily quickly. It is well known in control theory (see [3], [12], [14]) that this system is controllable if and only if the controllability matrix

$$
\left[\mathbf{b}|A \mathbf{b}| A^{2} \mathbf{b}|\cdots| A^{n-1} \mathbf{b}\right]
$$

has full rank $n$.

Generally, in control theory, no special structure or properties of the ma$\operatorname{trix} A$ or the vector $\mathbf{b}$ are assumed. However the matrix $A$ can be read as an adjacency matrix of a graph $G$ whose vertices are integrators (agents) and whose edges denote signal exchanges between the agents. In addition, $\mathbf{b}$ can be seen as a weighting term that describes the extent to which each agent is sensitive to a common external signal $u$. In particular, if all agents have the same sensitivity then we can take $\mathbf{b}=\mathbf{j}_{n}$. Then the matrix (2) is just the walk matrix of $G$, that is, the $n \times n$ matrix $W=\left(p_{i}(j-1)\right)$, where for any non-negative integer $k, p_{i}(k)$ denotes the number of walks of length $k$ starting at the $i$-th vertex of $G$.

In this context, the controllability is related to the main eigenvalues of the graph $G$, because $W$ has full rank $n$ if and only if $G$ has $n$ distinct main eigenvalues (see, for example, [16]).

We write $\mathbf{j}=\mathbf{j}_{n}$ and note in passing that a controllable graph is recognizable and reconstructible from its $n$-walk matrix

$$
W^{*}=\left(p_{i}(j)\right)=\left[A \mathbf{j}\left|A^{2} \mathbf{j}\right| A^{2} \mathbf{j}|\cdots| A^{n} \mathbf{j}\right] .
$$

For $W^{*}$ determines $W$, while $A W=W^{*}$; thus if $G$ is controllable then $A=$ $W^{*} W^{-1}$. Since $W^{*}=W C$, where $C$ is the companion matrix of the characteristic polynomial of $G$ (cf. [9]), we see that $G$ is reconstructible from its walk matrix and spectrum. A result of GODSIL and MCKAY [10] shows that a controllable graph is also reconstructible from its vertex-deleted subgraphs.

Other properties of controllable graphs may be found in $[\mathbf{9}],[\mathbf{1 1}],[\mathbf{1 7}]$; in particular, such graphs have a trivial automorphism group. This follows immediately from [15, Proposition 1.5]; alternative proofs are given in [7] and $[\mathbf{9}]$. Always a graph $G$ and its complement $\bar{G}$ have the same number of main eigenvalues (see, for example, [15, Proposition 1.3]). It follows that if $G_{1}, G_{2}$ are controllable graphs such that $\overline{G_{1}}, \overline{G_{2}}$ have disjoint spectra then their join (the complement of $\overline{G_{1}} \cup \overline{G_{2}}$ ) is also controllable. The trivial graph $K_{1}$ is controllable, while there are no other connected controllable graphs on fewer than 6 vertices. Further general observations and computational results concerning 
controllable graphs are given in [7]. It is conjectured there that almost all graphs are controllable; similar conjectures appear in [9] and [17].

We mention here certain configurations that are forbidden in controllable graphs. Recall that two non-adjacent vertices are said to be duplicate if they have the same neighbourhood, while coduplicate vertices are obtained by inserting an edge between a pair of duplicate vertices. We write $\sigma$ for the golden section $\frac{\sqrt{5}-1}{2} \approx 0.6180$.

Lemma 1.1. Controllable graphs do not contain

(i) a pair of duplicate vertices;

(ii) a pair of coduplicate vertices;

(iii) an induced path $P_{4}$ such that for any other vertex $x$ one of the following holds: (a) $x$ is adjacent to all vertices of $P_{4}$, (b) $x$ is adjacent to both terminal vertices of $P_{4},(\mathrm{c}) x$ is adjacent to both non-terminal vertices of $P_{4}$ or (d) $x$ is non-adjacent to any vertex of $P_{4}$.

Proof. Any pair of duplicate (resp. coduplicate) vertices gives rise to an eigenvector for the eigenvalue 0 (resp. -1 ) defined as follows: all its entries are zero except for entries 1 and -1 corresponding to these two vertices. Since such an eigenvector is orthogonal to the all-1 vector, statements (i) and (ii) follow.

Similarly, if some graph contains a path $P_{4}$ satisfying the specified conditions, we define an eigenvector as follows: let $1, \sigma,-\sigma$ and -1 be the entries corresponding to vertices of $P_{4}$ (in natural order), and let all other entries be equal to 0 . It is a matter of routine to verify that this eigenvector is orthogonal to the all-1 vector.

In the next section we provide some results on controllable graphs whose least eigenvalue is bounded from below by -2 . In Section 3 we note some consequences for controllable graphs whose second largest eigenvalue is bounded from above by 1. In Section 4 we observe that there is no non-trivial graph that is both integral and controllable, and we discuss controllability in the context of the signless Laplacian of a graph.

\section{CONTROLLABLE GRAPHS WITH EIGENVALUES BOUNDED FROM BELOW BY -2}

The line graph $L(G)$ is the graph whose vertices are the edges of $G$ with two vertices being adjacent whenever the corresponding edges are adjacent in $G$.

The cocktail party graph $C P(n)$ is the graph $\overline{n K_{2}}$. The generalized line graph $L\left(G ; a_{1}, \ldots, a_{n}\right)$ is defined for the graph $G$ with vertex set $\{1, \ldots, n\}$ and non-negative integers $a_{1}, \ldots, a_{n}$ by taking the graphs $L(G)$ and $C P\left(a_{i}\right)(i=$ $1, \ldots, n)$ and adding extra edges: a vertex $e$ in $L(G)$ is joined to all vertices in 
$C P\left(a_{i}\right)$ if $i$ is an endvertex of $e$ as an edge of $G$. Clearly, a generalized line graph reduces to a line graph whenever $a_{1}=\cdots=a_{n}=0$. If at least one of these parameters is greater than zero, the generalized line graph is called proper.

A connected graph is an exceptional graph if it is not a generalized line graph but has least eigenvalue not less than -2 .

The next classification can be found in [6]. The least eigenvalue of an arbitrary connected graph is greater than or equal to -2 if and only if it belongs to one of the following classes of graphs:

- the generalized line graphs;

- the exceptional graphs.

We first treat generalized line graphs.

Theorem 2.1. Controllable generalized line graphs are line graphs of either trees or odd unicyclic graphs.

Proof. By definition, any proper generalized line graph contains at least one pair of duplicate vertices (in some $C P\left(a_{i}\right)$ with $a_{i} \neq 0$ ), implying its noncontrollability. By [5, Theorem 2.6.4], -2 is never a main eigenvalue of a line graph. Finally, it is well-known (see, for example, [6, Theorem 2.3.20]) that connected line graphs with least eigenvalue greater than -2 are line graphs of either trees or odd unicyclic graphs.

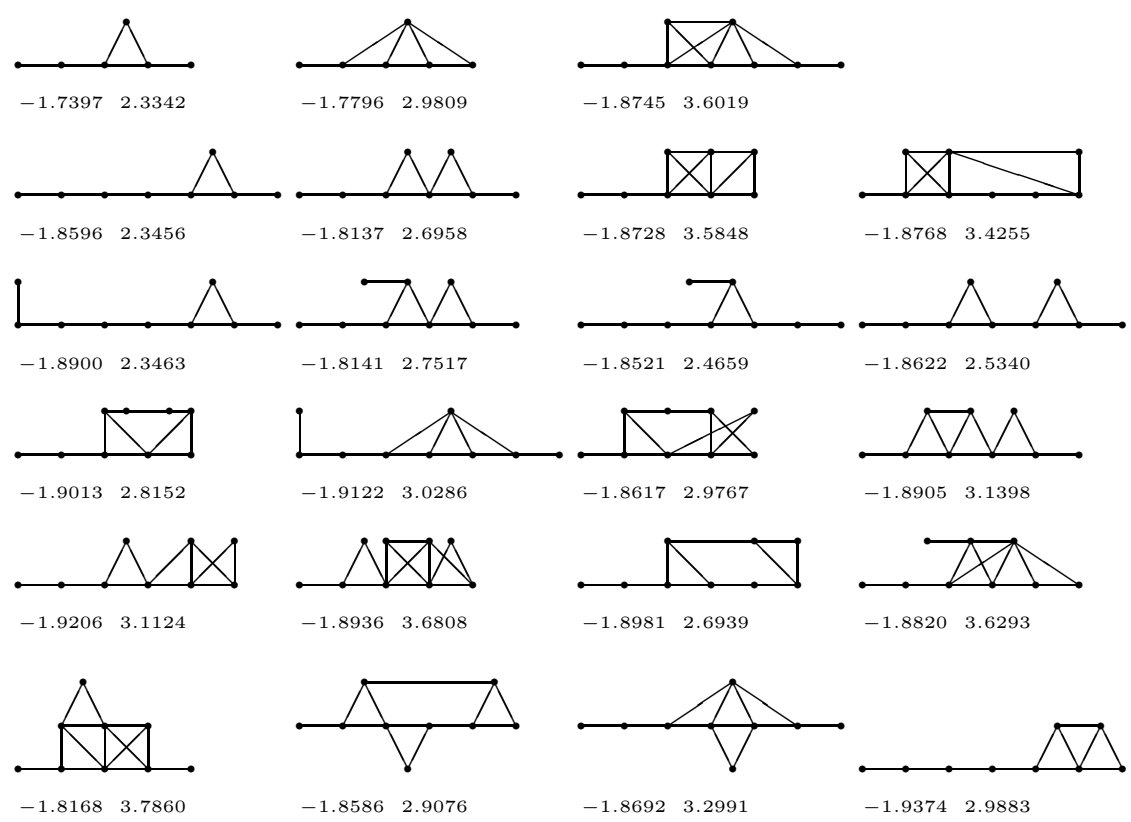

Figure 1. Controllable line graphs on 6,8 , and 9 vertices. 
In relation to the previous theorem, we note that the line graph of a tree or an odd unicyclic graph is not necessarily controllable. We found exactly 24 such graphs with 9 or fewer vertices. One of them is $K_{1}$, while the remaining graphs are depicted in Fig. 1 along with their least and largest eigenvalues; there are two graphs on 6 vertices, none on 7 vertices, three graphs on 8 vertices, and 18 graphs on 9 vertices.

To help us decide which line graphs of trees or of odd unicyclic graphs are controllable, we can study the structure of the corresponding trees and odd unicyclic graphs. For example, vertices of degree 1 cannot have a common neighbour since otherwise coduplicate vertices would appear in the line graph. Similarly, a triangle must have at least two vertices of degree greater than 2 . Some necessary spectral conditions are given in Section 4 (see Corollary 4.4 and Theorem 4.5).

Now we turn to exceptional graphs. Such graphs have at most 36 vertices, and any exceptional graph belongs to one of the following classes (see, for example, $[6])$ :

(i) the 573 exceptional graphs on $n$ vertices $(n=6,7,8)$ for which $\lambda(G)>-2$ (these graphs can be found in [6, Table A2]);

(ii) the exceptional graphs on $n=m+k$ vertices $(m=6,7,8)$ for which $\lambda_{m}>-2$ and $\lambda_{m+1}=\lambda_{m+2}=\cdots=\lambda_{m+k}=-2$ (these graphs are $k$-vertex extensions of the graphs from (i), and $\left.k \leq \frac{1}{2} m(m-1)\right)$.

Clearly, the exceptional controllable graphs whose least eigenvalue is greater than -2 have 6,7 or 8 vertices and all of them can be identified by considering the corresponding lists in $[6]$. In this way we can establish the following result.

Theorem 2.2. There are exactly 119 exceptional controllable graphs whose least eigenvalue is greater than -2 : two graphs have 6 vertices, 17 graphs have 7 vertices, and 100 graphs have 8 vertices. These graphs can be found in $[\mathbf{6}$, pp. 198-212] under the identifications given in Table 1.

The graph with 8 vertices identified in Table 1 by the number 50 is interesting since it is controllable, but also has a cospectral mate which is not controllable. Both graphs are depicted in Fig. 2. Their common spectrum is $3.0587,1.4263,0.6180,0.1901,-0.5164,-1.1803,-1.6180,-1.9783$, while 0.6180 and -1.6180 are the non-main eigenvalues of the second graph. Note that the first graph has a trivial and the second graph a non-trivial automorphism group. For spectral characterizations of controllable graphs, see [17].
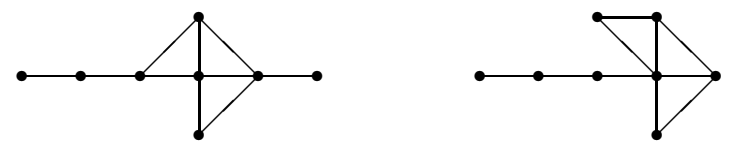

Figure 2. Controllable graph on 8 vertices and its non-controllable cospectral mate. 


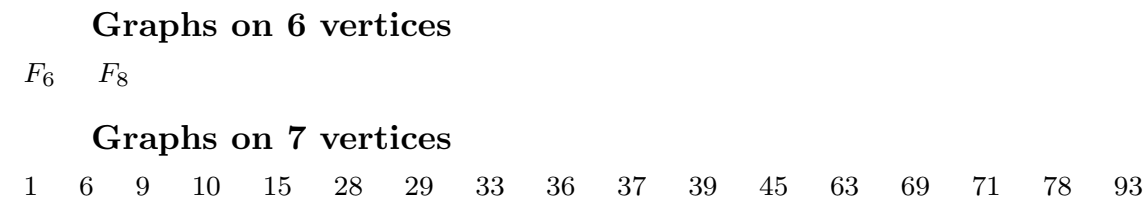

Graphs on 8 vertices

$\begin{array}{rrrrrrrrrrrrrrr}1 & 4 & 8 & 14 & 15 & 16 & 21 & 25 & 29 & 32 & 34 & 35 & 38 & 39 & 46 \\ 47 & 48 & 50 & 53 & 57 & 58 & 65 & 67 & 70 & 72 & 73 & 74 & 75 & 76 & 82 \\ 85 & 88 & 92 & 95 & 98 & 104 & 112 & 114 & 115 & 119 & 125 & 128 & 129 & 131 & 132 \\ 133 & 134 & 135 & 138 & 139 & 153 & 161 & 162 & 163 & 165 & 170 & 172 & 179 & 182 & 184 \\ 187 & 188 & 189 & 195 & 199 & 204 & 205 & 207 & 208 & 209 & 211 & 223 & 227 & 233 & 234 \\ 237 & 243 & 248 & 250 & 252 & 255 & 268 & 271 & 275 & 278 & 284 & 291 & 293 & 299 & 318 \\ 320 & 322 & 323 & 337 & 342 & 347 & 353 & 355 & 374 & 375 & & & & & \end{array}$

Table 1. Exceptional controllable graphs whose least eigenvalue is greater than -2 (the identifications are taken from from [6, Table A2]).

Theorem 2.3. There are exactly 587 exceptional controllable graphs whose least eigenvalue is equal to -2 : three graphs have 7 vertices, 45 graphs have 8 vertices, and 539 graphs have 9 vertices.

Proof. The graphs in question are one-vertex extensions of the exceptional graphs with least eigenvalue greater than -2 , for otherwise -2 is a multiple eigenvalue. Considering these graphs (some of which are listed in [6, Table A5]), we obtain the result by computer.

Amalgamating the results above we obtain the following theorem.

Thoerem 2.4. There are exactly 706 exceptional controllable graphs. Any other controllable graph with least eigenvalue at least -2 is the line graph of a tree or an odd-unicyclic graph.

\section{CONTROLLABLE GRAPHS WHOSE SECOND LARGEST EIGENVALUE DOES NOT EXCEED 1}

Using the results from the previous section and the well-known connections between $\lambda_{n}(\bar{G})$ and $\lambda_{2}(G)$, we investigate the controllable graphs $G$ for which $\lambda_{2}(G) \leq 1$. First we note that if $G$ is controllable then $\lambda_{2}(G)>\sigma$.

Theorem 3.1. There are no non-trivial controllable graphs satisfying $\lambda_{2} \leq \sigma$.

Proof. First, if a graph $G$ contains $P_{4}$ as an induced subgraph then its second largest eigenvalue is bounded from below by $\sigma\left(\right.$ since $\left.\lambda_{2}\left(P_{4}\right)=\sigma\right)$. If $\lambda_{2}(G)=\sigma$ then each vertex of $G$ either belongs to a fixed induced path $P_{4}$ or it satisfies one of the conditions given in Lemma 1.1 (iii) (see [8]). But then, by the same lemma, $G$ is not controllable.

Next, if $G$ does not contain $P_{4}$ as an induced subgraph then $\lambda_{2}(G)$ may or may not exceed $\sigma$, but in any case $G$ belongs to the well studied class of graphs 
known as cographs. Considering their structure (cf. [4]) we conclude easily that every non-trivial connected cograph contains at least one pair of duplicate or coduplicate vertices, and is therefore not controllable.

Now we give some results regarding controllable graphs satisfying $\lambda_{2} \leq 1$.

First, if the second largest eigenvalue of a graph $G$ does not exceed 1 then at most one eigenvalue of $\bar{G}$ is less than -2 (see [6, Theorem 7.1.1]). On the other hand, if $\lambda_{n}(G)>-2$ then $\lambda_{2}(\bar{G})<1$, while if $\lambda_{n}(G)=-2$ then $\lambda_{2}(\bar{G}) \leq 1$ with strict inequality if and only if -2 is a simple main eigenvalue of $G[6$, Theorem 7.1.2]). Now we can formulate the following result.

Theorem 3.2. Let $G$ be a controllable graph with $\lambda_{n}(G) \geq-2$, then $\bar{G}$ is controllable whenever it is connected. In addition, $\lambda_{2}(\bar{G})<1$.

Proof. The proof follows from the above arguments and the fact that a graph and its complement have the same number of main eigenvalues.

The controllable graphs with $\lambda_{2}<1$ whose complements satisfy $\overline{\lambda_{n}} \geq-2$ can be identified using Theorems 2.2 and 2.3. The remaining controllable graphs with $\lambda_{2} \leq 1$ will not be investigated here. We give only the following statistical data obtained by computer search: there are 4, 19, 168 and 925 controllable graphs satisfying $\lambda_{2} \leq 1$ with $6,7,8$ and 9 vertices, respectively.

Now we resolve one special case.

Theorem 3.3. There are exactly 25 controllable graphs satisfying $\lambda_{2} \leq 1$ and $\lambda_{n} \geq-2$. All of them are exceptional (16 graphs with $\lambda_{n}=-2$ and 9 graphs with $\lambda_{n}>-2$ ).

Proof. All the connected line graphs with $\lambda_{2} \leq 1$ are determined in [13, Theorem 3]: each is the induced subgraph of one of 8 graphs or of a graph in one infinite family. By inspection, no such graph is controllable: here we use the non-existence of controllable graphs on fewer than 6 vertices, the noncontrollability of line graphs which contain -2 as an eigenvalue, and the fact that any controllable graph has a trivial automorphism group.

Considering the exceptional graphs whose least eigenvalue is $\geq-2$ (Theorems 2.2 and 2.3) we obtain the assertion of the theorem.

In particular, if $\lambda_{n}=-2$ and $\lambda_{2} \leq 1$ then we obtain $0,2,3$, and 11 controllable graphs on $6,7,8$ and 9 vertices, respectively. If $\lambda_{n}>-2$ and $\lambda_{2} \leq 1$ then we obtain $2,4,3$, and 0 controllable graphs on $6,7,8$ and 9 vertices, respectively; their identifications in [6, Table A2] are: $F_{6}, F_{8}$ (6 vertices), 4,45 , 63, 78 (7 vertices) and 165, 227, 278, 284 (8 vertices).

So far we have not found any controllable graph whose second largest eigenvalue is equal to 1 .

\section{SOME OTHER RESULTS} results.

In this section we consider two further topics related to the foregoing 
A graph is called integral if its spectrum consists entirely of integers. A survey of results on integral graphs can be found in [2]. There are exactly 150 connected integral graphs on 10 or fewer vertices [1].

Theorem 4.1. The only integral controllable graph is the graph $K_{1}$.

We first prove a lemma.

Lemma 4.2. Let $G$ be a graph with $n$ vertices. If $G$ has $n$ distinct integer eigenvalues then $n \leq 10$.

Proof. Suppose that $G$ has $e$ edges and eigenvalues $\lambda_{1}, \ldots, \lambda_{n}$. If $n=2 k+1$ then $\Sigma_{i} \lambda_{i}^{2}$ is at least $2\left(1^{2}+2^{2}+\cdots+k^{2}\right)$ (corresponding to a spectrum $k$, $k-1, \ldots, 1,0,-1,-2, \ldots,-k)$. Therefore

$$
2 k(2 k+1) \geq 2 e=\Sigma_{i} \lambda_{i}^{2} \geq \frac{1}{3} k(k+1)(2 k+1),
$$

whence $k \leq 5$. If $k=5$ then $e=55$ and $G=K_{11}$, a contradiction. Hence $n \leq 9$ when $n$ is odd.

$$
\begin{aligned}
& \text { If } n=2 k \text { then } \\
& \qquad \Sigma_{i} \lambda_{i}^{2} \geq k^{2}+2\left(1^{2}+2^{2}+\cdots+(k-1)^{2}\right) .
\end{aligned}
$$

In fact, this inequality is strict because there is no spectrum $k, \ldots, 1,0,-1, \ldots$, $-(k-1)$ and no spectrum $k-1, \ldots, 1,0,-1, \ldots-k$. Hence

$$
2 k(2 k-1) \geq k^{2}-1+\frac{1}{3}(k-1) k(2 k-1)=\frac{1}{3}(k-1)\left(2 k^{2}+2 k+3\right),
$$

and we have $k \leq 5, n \leq 10$.

Now Theorem 4.1 is easily verified by inspecting the spectra of the 150 connected integral graphs with 10 or fewer vertices [1].

In the second topic we make use of the main angles and the signless Laplacian of a graph, defined as follows. For an arbitrary graph $G$ with $n$ vertices and distinct eigenvalues $\mu_{1}, \ldots, \mu_{m}$, the main angle $\beta_{i}$ is the cosine of the angle between $\mathbf{j}_{n}$ and the eigenspace of $\mu_{i}(i=1, \ldots, m)$. For the role of main angles in a graph, see [15] or [5, Section 4.5]. If $G$ has adjacency matrix $A$, the signless Laplacian matrix of $G$ is the matrix $Q=D+A$, where $D$ is the diagonal matrix of vertex degrees. We say that a connected graph with $n$ vertices is $Q$-controllable if its signless Laplacian $Q$ has no eigenvector orthogonal to $\mathbf{j}_{n}$.

We prove the following result.

Theorem 4.3. A tree $T$ with $n$ vertices is $Q$-controllable if and only if $L(T)$ is controllable and $T$ is not a spanning subgraph of $K_{r, r}($ where $n=2 r)$.

Proof. Let $T$ have adjacency matrix $A$ and vertex-edge incidence matrix $B$ (of size $n \times(n-1))$. Then $L(T)$ has adjacency matrix $B^{\top} B-2 I$, while $Q=B B^{\top}$. We exploit the fact that $B B^{\top}$ and $B^{\top} B$ have the same non-zero eigenvalues. 
If $L(T)$ is controllable then $\lambda_{n-1}(T)>-2$ and so $B^{\top} B$ has distinct positive eigenvalues, say $\xi_{1}, \ldots, \xi_{n-1}$ where $\xi_{i}=\lambda_{i}(L(T))+2(i=1, \ldots, n-1)$. Then the eigenvalues of $B B^{\top}$ are $0, \xi_{1}, \ldots, \xi_{n-1}$. On the other hand, if $T$ is $Q$ controllable, then the eigenvalues of $B B^{\top}$ are $0, \xi_{1}, \ldots, \xi_{n-1}$, where the distinct positive numbers $\xi_{1}, \ldots, \xi_{n-1}$ are the eigenvalues of $B^{\top} B$.

Let $\mathbf{x}_{0}, \mathbf{x}_{1}, \ldots, \mathbf{x}_{n-1}$ be eigenvectors of $B B^{\top}$ corresponding to $0, \xi_{1}, \ldots$, $\xi_{n-1}$, respectively. Then $B^{\top} \mathbf{x}_{1}, \ldots, B^{\top} \mathbf{x}_{n-1}$ are eigenvectors of $B^{\top} B$ corresponding to $\xi_{1}, \ldots, \xi_{n-1}$. We may scale $\mathbf{x}_{1}, \ldots \mathbf{x}_{n-1}$ so that

$$
\mathbf{j}_{n-1}=\beta_{1} B^{\top} \mathbf{x}_{1}+\cdots+\beta_{n-1} B^{\top} \mathbf{x}_{n-1},
$$

where $\beta_{1}, \ldots, \beta_{n-1}$ are the main angles of $L(T)$. Let

$$
\mathbf{j}_{n}=\gamma_{0} \mathbf{x}_{0}+\gamma_{1} \mathbf{x}_{1}+\cdots+\gamma_{n-1} \mathbf{x}_{n-1} .
$$

Since $B^{\top} \mathbf{x}_{0}=\mathbf{0}$, we have

$$
B^{\top} \mathbf{j}_{n}=\gamma_{1} B^{\top} \mathbf{x}_{1}+\cdots+\gamma_{n-1} B^{\top} \mathbf{x}_{n-1} .
$$

Since $B^{\top} \mathbf{j}_{n}=2 \mathbf{j}_{n-1}$, we have $\gamma_{i}=2 \beta_{i}(i=1, \ldots, n-1)$. Hence $T$ is $Q$ controllable if and only if $\gamma_{0} \neq 0$ and all of $\beta_{1}, \ldots, \beta_{n-1}$ are non-zero.

Now $T$ is a spanning subgraph of some $K_{p, q}(p \leq q)$, where $p+q=n$ and $p, q$ are determined uniquely by $T$. With appropriate labelling of the vertices of $T, A$ has the form $\left(\begin{array}{cc}O & E^{\top} \\ E & O\end{array}\right)$; then we may take $\mathbf{x}_{0}=\left(\begin{array}{c}\mathbf{j}_{p} \\ -\mathbf{j}_{q}\end{array}\right)$ because $\left(\begin{array}{c}\mathbf{j}_{p} \\ \mathbf{j}_{q}\end{array}\right)$ spans the 0-eigenspace of $D-A$ (the Laplacian). Hence $\mathbf{j}_{n}^{\top} \mathbf{x}_{0}=0$ if and only if $p=q$. Hence $T$ is $Q$-controllable if and only if $L(T)$ is controllable and $T$ is not a spanning tree of $K_{r, r}$ (where $r=p=q$ ).

From the above proof, we can deduce the following.

Corollary 4.4. Let $T$ be a tree with $n$ vertices. If $L(T)$ is controllable with eigenvalues $\lambda_{1}, \ldots, \lambda_{n-1}$ and corresponding main angles $\beta_{1}, \ldots, \beta_{n-1}$, then

$$
n \geq 4(n-1)\left(\frac{\beta_{1}^{2}}{\lambda_{1}+2}+\cdots+\frac{\beta_{n-1}^{2}}{\lambda_{n-1}+2}\right),
$$

with equality if and only if $T$ is a spanning tree of $K_{r, r}$ (where $\left.n=2 r\right)$.

Proof. Let $\xi_{i}=\lambda_{i}+2(i=1, \ldots, n-1)$. Note first that, from Equation (3), we have $\left\|B^{\top} \mathbf{x}_{i}\right\|=\sqrt{n-1}$ because $\left\|B^{\top} \mathbf{x}_{i}\right\|\left\|\mathbf{j}_{n-1}\right\| \beta_{i}=\beta_{i}\left\|B^{\top} \mathbf{x}_{i}\right\|^{2}$. Now $\mathbf{x}_{i}^{\top} B B^{\top} \mathbf{x}_{i}=\xi_{i}\left\|\mathbf{x}_{i}\right\|^{2}$, and so $\left\|\mathbf{x}_{i}\right\|^{2}=(n-1) / \xi_{i}(i=1, \ldots, n-1)$.

Now $\left\|\mathbf{j}_{n}\right\|^{2}=\gamma_{0}^{2}\left\|\mathbf{x}_{0}\right\|^{2}+4 \beta_{1}^{2}\left\|\mathbf{x}_{1}\right\|^{2}+\cdots+4 \beta_{n-1}^{2}\left\|\mathbf{x}_{n-1}\right\|^{2}$, whence $n \geq$ $4(n-1)\left(\frac{\beta_{1}^{2}}{\xi_{1}}+\cdots+\frac{\beta_{n-1}^{2}}{\xi_{n-1}}\right)$, with equality if and only if $\mathbf{j}_{n}$ is orthogonal to $\mathbf{x}_{0}$. The result follows.

Using similar arguments, we can prove the following. 
Theorem 4.5. An odd-unicyclic graph $U$ is $Q$-controllable if and only if $L(U)$ is controllable. Moreover, if $L(U)$ is controllable with eigenvalues $\lambda_{1}, \ldots, \lambda_{n}$ and corresponding main angles $\beta_{1}, \ldots, \beta_{n}$ then

$$
\left(\frac{\beta_{1}^{2}}{\lambda_{1}+2}+\cdots+\frac{\beta_{n}^{2}}{\lambda_{n}+2}\right)=\frac{1}{4}
$$

Aknowledgements. The work is supported by the Serbian Ministry of Education and Science, Projects 174012 and 174033.

\section{REFERENCES}

1. K. T. Balińska, D. Cvetković, M. Lepović, S. K. Simić: There are exactly 150 connected integral graphs up to 10 vertices. Univ. Beograd, Publ. Elektrotehn. Fak. Ser. Mat., 10 (1999), 95-105.

2. K. T. Balińska, D. Cvetković, Z. Radosavljević, S. K. Simić, D. SteVANOvić: A survey on integral graphs. Univ. Beograd, Publ. Elektrotehn. Fak. Ser. Mat., 13 (2002), 42-65; Errata, op. cit. 15 (2004), 112.

3. C. T. Chen: Linear System Theory and Design. Oxford University Press Inc, Evans Road Cary, NC, 1984.

4. D. G. Corneil, Y. Perl, L. K. Stewart: A linear recognition algorithm for cographs. SIAM J. Comput., 14 (1985), 926-934.

5. D. Cvetković, P. Rowlinson, S. Simić: Eigenspaces of Graphs. Cambridge University Press, Cambridge, 1997.

6. D. Cvetković, P. Rowlinson, S. Simić: Spectral Generalizations of Line Graphs - On Graphs with Least Eigenvalue -2. London Math. Soc., Lecture Note Series 314, Cambridge University Press, Cambridge, 2004.

7. D. Cvetrović, P. Rowlinson, Z. Stanić Z., M. G. Yoon: Controllable graphs. Bull. Acad. Serbe Sci. Arts. Classe Sci. Math. Natur. Sci. Math., 140 (36) (2011), to appear.

8. D. Cvetković, S. Simić: On graphs whose second largest eigenvalue does not exceed $\frac{\sqrt{5}-1}{2}$. Discrete Math., 138 (1995), 213-227.

9. C. Godsil: Controllable subsets in graphs. ArXiv: 1010.3231v1 [math.CO] 15 Oct. 2010.

10. C. Godsil, B. D. McKaY: Spectral conditions for the reconstructibility of a graph. J. Combin. Theory Ser. B, 30 (1981), 285-289.

11. C. Godsil, S. Severini: Control by quantum dynamics on graphs. Phys. Rev. A, 81, 052316(2010).

12. T. Kailath: Linear Systems. Prentice-Hall, Inc., Englewood Cliffs, N.J., 1980.

13. M. Petrović, B. Milekić: On the second largest eigenvalue of line graphs. J. Graph Theory 27(1998), 61-66.

14. A. Rahmani, M. Ji, M. Mesbahi, M. Egerstedt: Controllability of multiagent systems from a graph-theoretic perspective. SIAM J. Control. Optim., 48 (1) (2009), 162-186. 
15. P. Rowlinson: The main eigenvalues of a graph: A survey. Appl. Anal. Discrete Math., 1 (2) (2007), 455-471.

16. Y. Teranishi: Main eigenvalues of a graph. Linear Multilinear Algebra, 49 (2002), 289-303.

17. W. WAnG, C. XU: A sufficient condition for a family of graphs being determined by their generalized spectra. European J. Combin., 27 (6) (2006), 826-840.

Mathematical Institute SANU,

(Received August 5, 2011)

P.O. Box 367,

11000 Belgrade,

(Revised September 9, 2011)

Serbia

E-mail: ecvetkod@etf.rs

Institute of Computing Science and Mathematics, University of Stirling,

Stirling FK9 4LA,

Scotland, UK

E-mail: p.rowlinson@stirling.ac.uk

Faculty of Mathematics,

University of Belgrade,

11000 Belgrade,

Serbia

E-mail: zstanic@math.rs

Department of Precision Mechanical Engineering,

Gangneung-Wonju National University,

Gangnueng 120-702,

Republic of Korea

E-mail: mgyoon@gwnu.ac.kr 